\title{
Bilateral Symptomatic Lateral Parapatellar Synovial Plica of the Knee in an Adolescent Athlete: A Case Report
}

\author{
Hiroaki Kanazawa1 ${ }^{*}$, Yuichiro Maruyama1, Shigemasa Takamiya ${ }^{1}$, Kazuo Kaneko ${ }^{2}$ \\ ${ }^{1}$ Department of Orthopaedic Surgery, Juntendo University Urayasu Hospital, Chiba, Japan \\ ${ }^{2}$ Department of Orthopaedic Surgery, Graduate School of Medicine, Juntendo University, Tokyo, Japan \\ Email: *hiroakimed@yahoo.co.jp
}

How to cite this paper: Kanazawa, H., Maruyama, Y., Takamiya, S. and Kaneko, K. (2017) Bilateral Symptomatic Lateral Parapatellar Synovial Plica of the Knee in an Adolescent Athlete: A Case Report. Open Journal of Orthopedics, 7, 63-71. https://doi.org/10.4236/ojo.2017.72008

Received: January 12, 2017

Accepted: February 10, 2017

Published: February 13, 2017

Copyright (c) 2017 by authors and Scientific Research Publishing Inc. This work is licensed under the Creative Commons Attribution International License (CC BY 4.0).

http://creativecommons.org/licenses/by/4.0/

\begin{abstract}
We report herein a rare case of lateral parapatellar synovial plica that developed simultaneously in both knees. A 15-year-old competitive soccer player visited our institution with a six-month history of anterolateral pain and catching sensation in both knee joints. On physical examination, he complained of tenderness along the joint line of bilateral patellofemoral joint (PFJ). Visible and palpable popping was observed at the lateral margin of each patella during active knee motion. Magnetic resonance imaging showed obvious synovial soft nodules in the lateral side of the PFJ. On arthroscopy, a yellowish, thick, tongue-shaped tissue extending transversely from the lateral parapatellar synovium was identified in both knees, and parts of this tissue showed avascular hypertrophy. Arthroscopic findings of both knees are almost symmetrical in anatomical location, but no similar in size. After arthroscopic excision, the patient became asymptomatic. At 24-month follow up, he demonstrated full knee function, without evidence of local recurrence.
\end{abstract}

\section{Keywords}

Lateral Parapatellar Synovial Plica, Impingement of Patellofemoral Joint (PFJ), Anterior Knee Pain

\section{Introduction}

Synovial plica or fold is some of the normal structures of the knee joint cavity, and is basically asymptomatic. When synovial plica loses their normal elasticity and becomes fibrotic, they can be cause of dynamic pathological lesion of the knee. The medial parapatellar plica is most common symptomatic, and little lateral parapatellar plica has been published and is considered a much more rare 
entity (1\% to $2 \%)$ [1] [2].

Furthermore, bilateral symptomatic lateral parapatellar plica occurring simultaneously during the adolescent growth period is not found reported in the literature. We present hereinafter a rare case of lateral parapatellar synovial plica involving bilateral patello-femoral joint (PFJ) with restriction of sports activity and severe pain.

\section{Case Report}

A 15-year-old competitive soccer player visited our institution with a six month history of pain and catching sensation in both knee joints. He collided with another player during a soccer game and was injured on the right knee in December, 2013. At first, he complained knee pain in right side. Thereafter, he complained symptom on opposite side. Knee pain continued after the initial collision and he began to feel a catching, popping sensation in March, 2014. There was no history of any other previous trauma.

The catching and pain symptoms were aggravated by motions such as ascending or descending stairs, squatting, and after prolonged sports activity. At the initial consultation, iliotibial tract friction syndrome was diagnosed, and physical therapy was tried for six months in another orthopaedics clinic, but the symptoms were not relieved. Therefore, the patient visited our department to get a second opinion in July, 2014.

He was suffering from episodes of anterolateral knee pain and catching with apprehension in both knee joints. There was no history of intra-articular injection of steroids or hyaluronic acid to the knee joint. Body weight was $61.7 \mathrm{~kg}$ and height was $172 \mathrm{~cm}$. There were no abnormal laboratory results.

At the initial physical examination, there was localized tenderness at the lateral and inferior borders of the bilateral patella. Visible and palpable popping was observed at the lateral border of each patella during active knee motion, from flexion to extension. A palpable nodule could be felt moving over the femoral condyle during active knee motion, with flexion at approximately 30 degrees, but popping was not recognized with passive knee motion. There was slight knee effusion or swelling in the right knee, but not in the left knee. Mobility, tracking of the patella on the patellar groove, was normal. The range of motion was normal with no instability. The International Knee Documentation Committee (IKDC) knee examination form was $\mathrm{C}$ and the Lysholm knee score was 64 in both knee.

Preoperative Merchant view radiographs of both knee joints showed a slight lateral tilt of the patella. The quadriceps angle ( $\mathrm{Q}$ angle) was normal. The standing femorotibial angle (FTA) was 177 degrees.

Preoperative magnetic resonance imaging (MRI) on T2 proton densityweighted (PDW) images (TR/TE:1924/18) showed synovial nodules of an intermediate intensity signal in the lateral side of the bilateral patello-femoral joint (PFJ) [Figure 1(a) and Figure 1(b)].

MRI findings of both knees are almost symmetrical in location, but no similar 


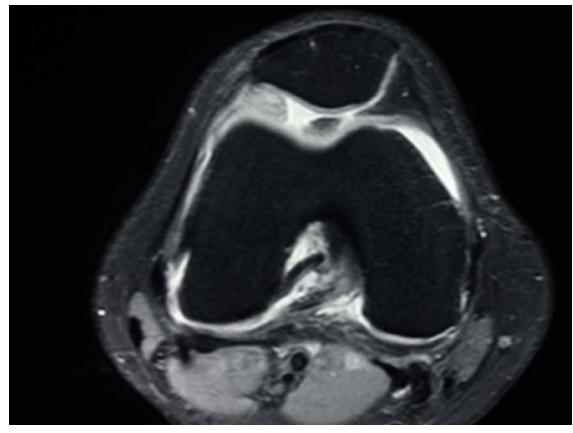

(a)

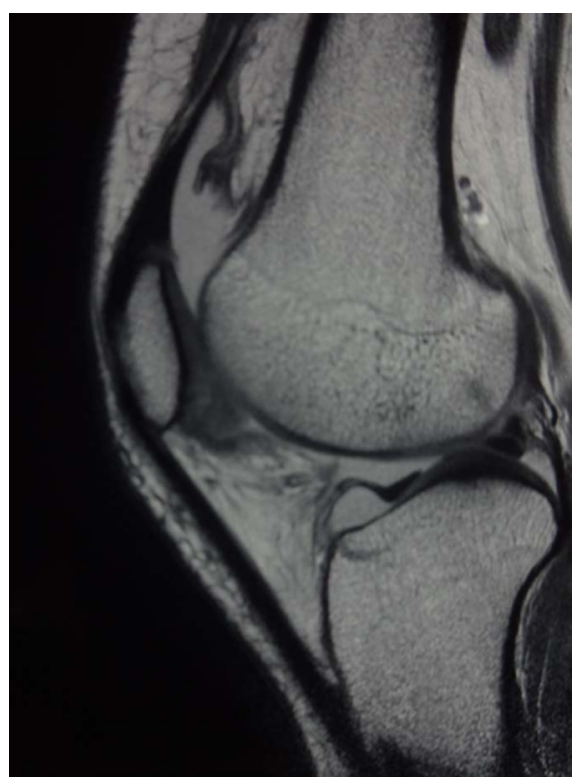

(c)

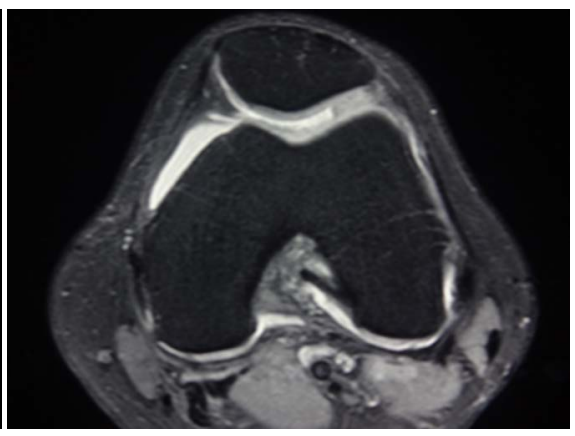

(b)

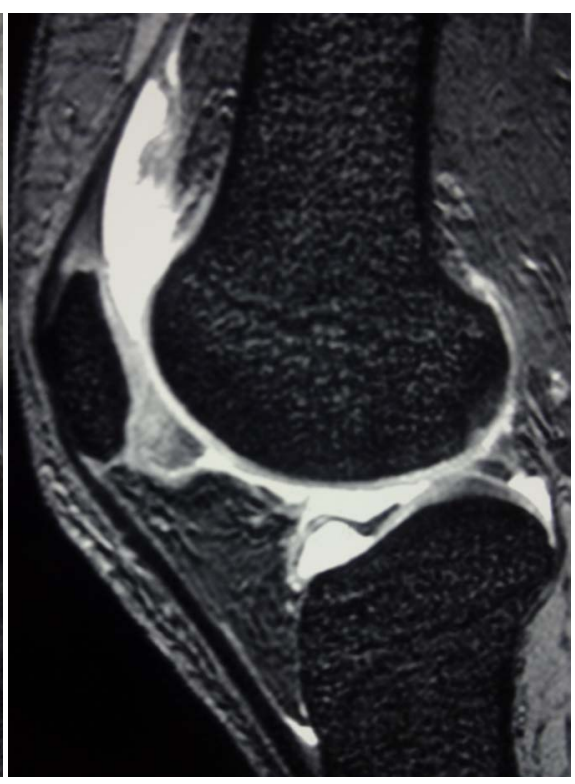

(d)

Figure 1. ((a), (b)) Axial proton density-weighted images MRI (TR/TE:1924/18) showed synovial tissue of intermediate intensity signal in the lateral side of the patellofemoral joint. (a) right knee, (b) left knee; ((c), (d)) On saggital view, a cartilage gradient echo (TR/TE:19/7) MRI shows synovial soft tissue, low intensity signal, and a T2 proton density-weighted (PDW) image (TR/TE:3000/30) shows intermediate intensity signal located on the lateral side of the patellofemoral joint.

in size.

On the saggital view, a cartilage gradient echo (TR/TE:19/7) MRI scan showed low intensity signal, and a T2 PDW image (TR/TE:3000/30) showed an intermediate intensity signal at the same location [Figure 1(c) and Figure 1(d)].

We did not perform intra-articular and intra-plical injections of steroids or hyaluronate. Conservative therapy consisting of physical therapy, activity modification and non-steroid anti-inflammatory medications had failed for at least 6 months. Therefore, we considered surgical intervention and performed arthroscopy of both knees in December 2014.

Under bilateral knee arthroscopy, no medial, lateral meniscus tears or loose bodies, or osteochondritis dissecans were found. The ACL and PCL were intact. In the right knee, a yellowish, thick, tongue-shaped tissue extending transversely from the lateral parapatellar synovium, that covers part of the lateral patellar fa- 
cet, was visualized arthroscopically, and parts of this tissue showed avascular hypertrophy [Figure 2(a)]. Irregularities on the edge of chondral surfaces of the lateral femoral condyle [Figure 2(c)] were seen in the right knee. In contrast, a yellowish-whitish, avascular hypertrophic, synovial plica was observed in the lateral parapatellar area of the left knee, and palpation of the plica with a probe confirmed the thick nodule [Figure 2(b)]. The type of bilateral plica was the almost similar in shape, but slightly smaller in left side.

On arthroscopy, the synovial fold impinged into the bilateral side of the PFJ during knee extension from the flexed position. Therefore, the hypertrophied tissues were debrided with a combination of a punch and an electrocautery abrasion device (VAPR system, DePuy Mitek, USA), and the plica was removed from each knee. Meticulous haemostasis by electrocautery following tourniquet release was done to avoid postoperative hemarthrosis.

Histologically, examination of the resected synovial tissue revealed a mild papillary proliferation, fibrosis of synovial tissue, accompanied by a mild lymphocytic infiltration with fatty tissue [Figure 3(a) and Figure 3(b)].

Full weight bearing was allowed just after the operation for both knee joints. Passive and active range of motion of the knee was started immediately after the operation. There were no complications affecting the results except postoperative hemarthrosis, due to surgical intervention, which required aspiration once

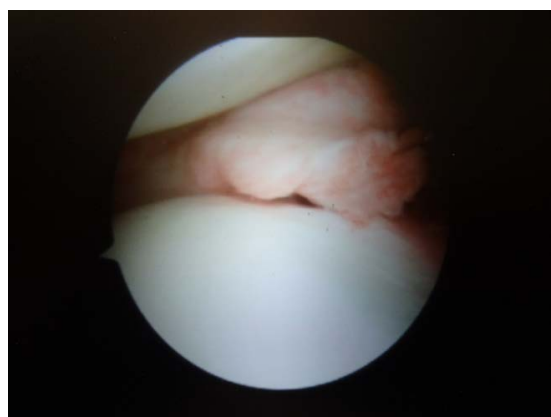

(a)

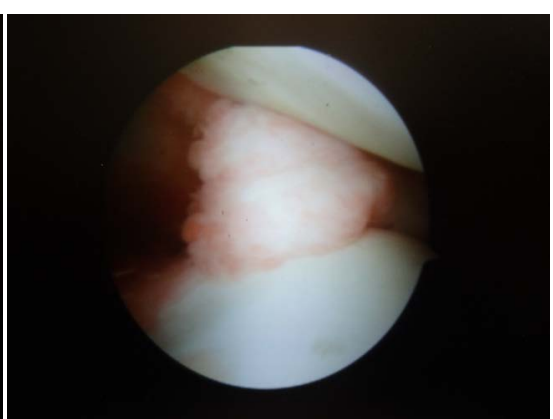

(b)

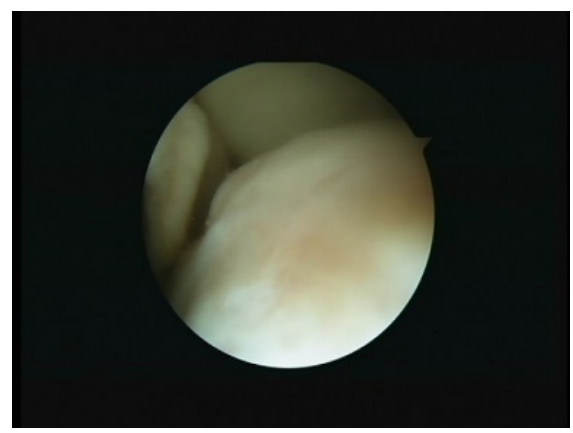

(c)

Figure 2. ((a), (b)) In both knee, a yellowish, thick, tongue-shaped tissue extending transversely from the lateral parapatellar synovium that covers part of the lateral patellar facet is visualized arthroscopically, and parts of this tissue show avascular hypertrophy. The type of bilateral plica was the almost similar in shape, but slightly smaller in left side. (c) Irregularities on the edge of chondral surfaces of the lateral femoral condyle are shown in the right knee. 


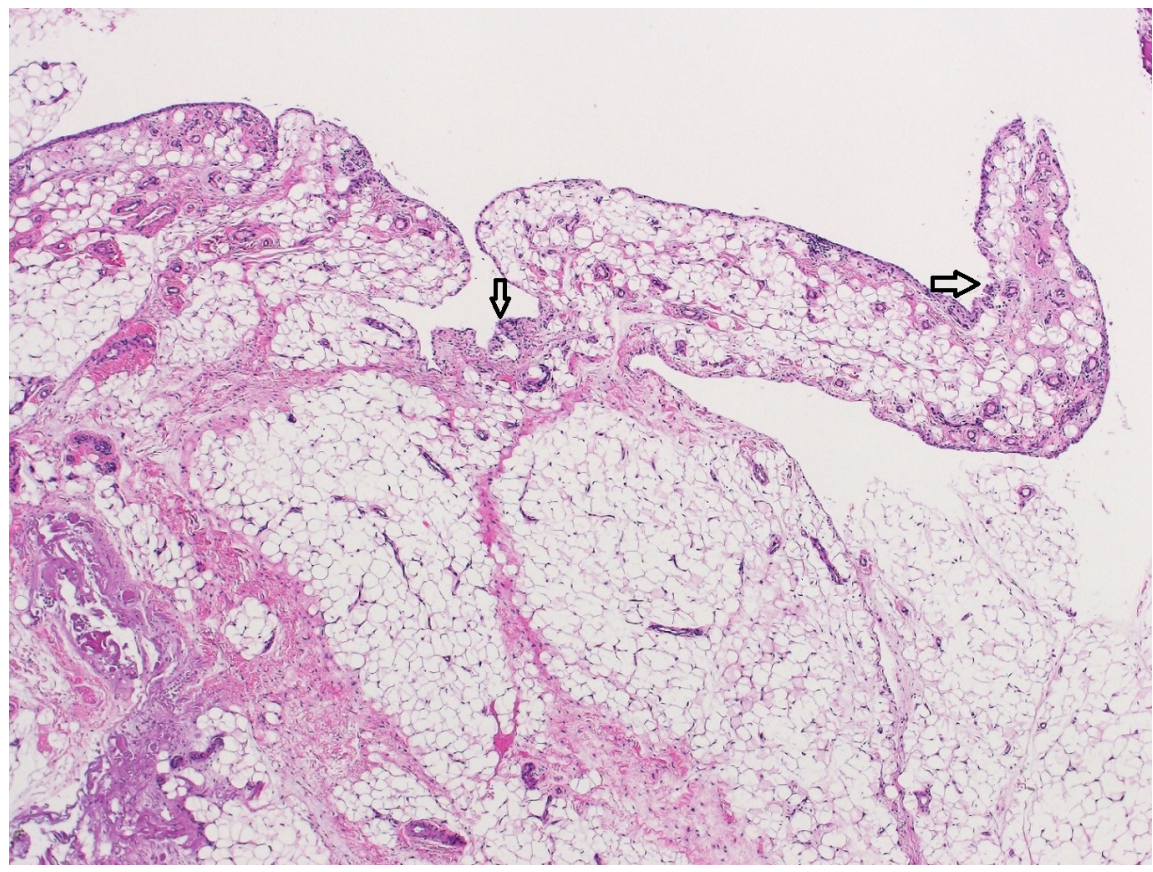

(a)

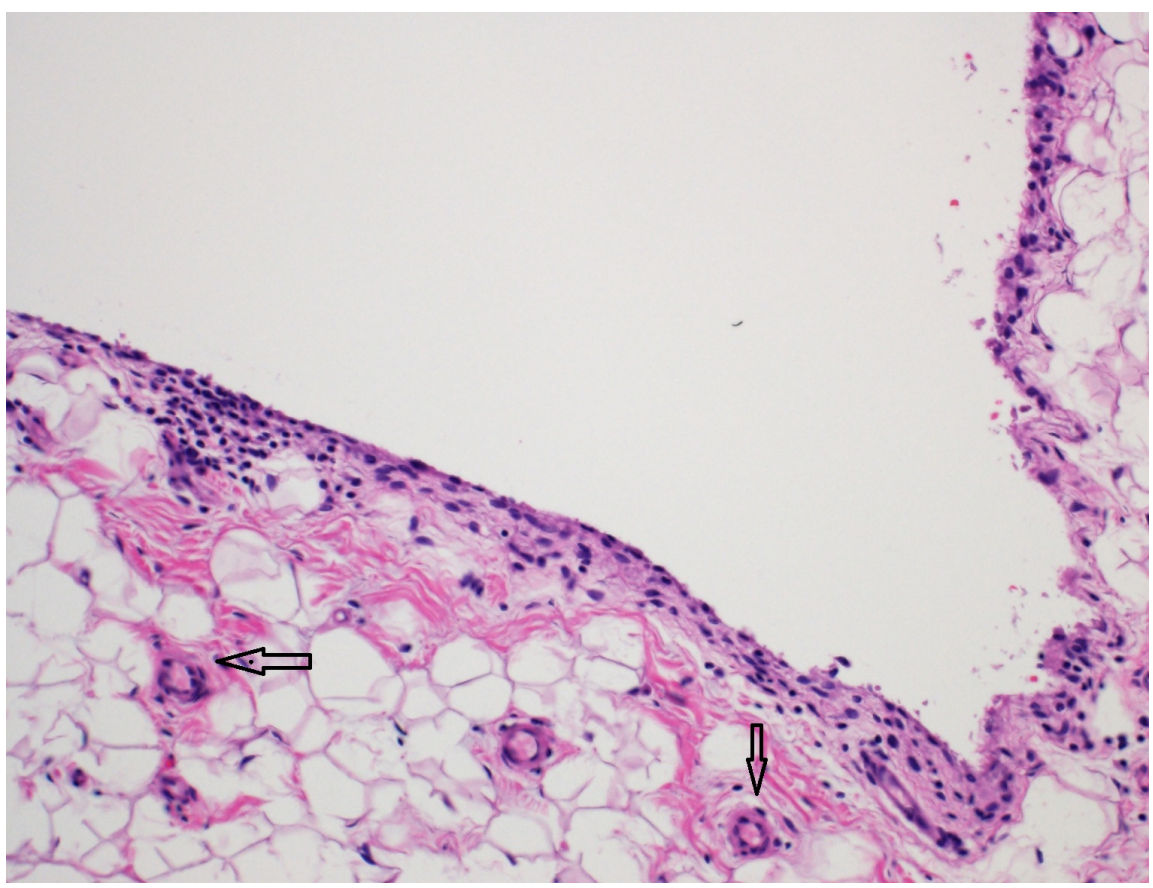

(b)

Figure 3. Fibrous tissue lined with synovial membrane is seen in; (a) section (Hematoxylin-eosin stain, original magnification $\times 40$ ), (b) section (Hematoxylin-eosin stain, original magnification $\times 200$ ).

in the right knee. After arthroscopic excision of the bilateral knee, the preoperative symptoms disappeared gradually. The patient had a full range of pain-free motion with no impingement to daily life. The patient returned to regular soccer three months after surgery. One year after surgery, the patient was asymptomatic and had completely returned to sports activity. At a recent examination 24 
months later, the IKDC knee examination form were A in both knees and IKDC subjective knee evaluation form were 96.6 for the right side, 98.9 for the left side. The Lysholm knee score were 99 points for the right side and 100 points for the left side.

\section{Discussion}

Synovial plica (fold) around the knee is common in arthroscopy, but rarely pathological. Plicae are usually thin and elastic connective tissues that are harmless and unobstructive. However, plicae can become thickened and inelastic with inflammatory processes of the synovium, including hemarthrosis sencodary to trauma and mechanical irritation from repetitive sports activity. As a consequence, these tissues can damage nearby intraarticular structures, a process called pathologic synovial plica syndrome. Synovial plica syndrome is a wellestablished cause of anterior knee pain in some patients.

The important findings of this report showed that extremely rare lateral parapatellar synovial plica developed simultaneously in both knees by one trauma, and which occurred during the adolescent growth period. This was the unique part of our case which differs from the previous cases regarding bilateral synovial lateral plica.

It can be difficult to diagnose whether plicae are symptomatic even if synovial plica is found in preoperative MRI. Conversely, MRI is often non-diagnostic even when plicae are suspected clinically. Regarding radiological diagnosis, the paper published by Kobayashi et al. [3] reported reliability of the MRI arthrography for accurate diagnosis of medial plica is significantly higher than conventional MRI.

As differential diagnosis, meniscus tears or loose bodies, or osteochondritis dissecans were considered. Differentiating synovial plica syndrome from other more common causes of knee pain is difficult and it is only with a careful review of history and examination that suspicions are raised, but the pathological nature and the necessity for treatment is controversial.

Traditionally, plicae have been divided into four types based on their anatomical location; the infrapatellar or ligamentum mucosum, the suprapatellar, the mediopatellar or the medial shelf, and lateral patellar or lateral synovial plica [4]. The orthopaedic literature reports that infrapatellar plica (IPP) and mediopatellar plica (MPP) are frequently encountered during arthroscopic surgery. In these types, the most common symptomatic plica, which causes soft tissue impingement syndrome in the PFJ, are the MPP, but the prevalence of lateral parapatellar plica (LPP) ranges from around $1 \%$ to $2 \%$, which is considered rather rare [1] [2].

A thorough review of orthopaedic literature revealed only a few reported cases of symptomatic lateral synovial plica syndrome [2] [5] [6]. Kim and Choe described it in their report on 400 arthroscopies, and classified lateral plicae into three types; absent, shelf and fenestra [7]. They found an overall incidence of 1\%. Older et al. found 1 incidence in 115 knees [8]. Tearse et al. encountered 21 
significant lateral plicae in 3000 arthroscopies (0.7\%), and estimated that 14 of them were fibrotic and symptomatic [9].

Furthermore, bilateral synovial lateral lesions are extremely rare. To the best of our knowledge, there are only two publications regarding bilateral synovial lateral plica syndrome occurring simultaneously [1] [10]. In previous two report cases, the age of onset were 37 and 21 years. In contrast, symptom was occurred during adolescent growth period in our case. A thorough review of orthopaedic literature revealed no prior reports of bilateral synovial lateral plica occurring simultaneously during the adolescent growth phase.

Dupont et al. described four different synovial structures in the lateral gutter; classified into the lateral patellar plica, lateral alar fold, superolateral fold and transverse synovial arcuate fold [4]. Knee pain stemming from these structures may be missed or incorrectly diagnosed, and these patients often present for second or third opinions because of failure to diagnose and treat the underlying pathology.

In previous literature as a factor associated with incidence, Broom and Fulkerson mentioned that $41 \%$ of patients with painful plicae had no history of trauma and most had no inflammation [11]. In most cases, symptoms were triggered by a stimulatory event. Schindler reported that trauma was considered the cause in approximately $50 \%$ of cases [12]. Interestingly, a symptom occurred in the opposite knee at the same time in our case.

There was no history of trauma in previous two reports regarding bilateral synovial lateral plica [1] [10]. One key point for consideration in this case is that whether left knee injury is post-traumatic or constitutional or combined or not. It was unclear that why symptoms were occurred in bilateral knee by only one trauma.

It was thought that the likely mechanism of the injury was a combination of directional force on the right knee joint, together with the twisting injuries on the left knee joint. As a result, an inflammatory process secondary to acute blunt trauma, mechanical irritation from repetitive sports activity, may be related to anatomic change within the bilateral knee and the plica itself. Furthermore, patient was a 15 -years-old boy in the adolescent growth period. We speculate that the etiology of our case may be that an adolescent growth cycle in which the less compliant lateral patellar soft tissues become a primary restraint to the PFJ with growth of the distal femoral epiphysis, and then direct injury to the knee and mechanical irritation from athletic sports activity induces reactive synovitis. Furthermore, when he was kicking ball as a soccer player, both knees generally assumed a valgus position, and repetitive flexion and extension motion. This is also a possible cause of the injury to the plica and the joint cartilage.

Conservative therapy is the initial treatment strategy. Any conservative therapy should include a period of rest from sporting and strenuous physical activities, nonsteroidal anti-inflammatory drugs, quadriceps strengthening, hamstirngs stretching, and corticosteroid injections. However, success rates of these therapy are variable, and complete relief of symptoms is seldom if ever achievable. 
Patients who do not respond to conservative therapy or have recurrence of symptoms are essentially the candidates for arthroscopic surgery of the knee. In a recent meta-analysis that included 969 patients treated either with open or arthroscopic plica resection at a mean follow-up of 2 years, $64 \%$ of patients were free of symptoms and returned to unlimited activities, $26 \%$ had occasional symtoms that did not affect their activity, and $10 \%$ did not show improvement or experienced worsening of symproms [12].

\section{Conclusion}

In summary, physician should keep in mind that lateral synovial plica should be considered as a potential, sole cause of anterior knee pain with probable MRI imaging and no other evidences of internal pathological lesion. A patient with extremely rare lateral parapatellar synovial plica, which affected simultaneously in both knees of adolescent athlete, is reported.

\section{Consent}

We received written informed consent from the patient for publication of this article.

\section{Conflicts of Interest}

No conflicts of interest were declared by the authors.

\section{References}

[1] Kurosaka, M., Yoshiya, S., Yamada, M. and Hirohata, K. (1992) Lateral Synovial Plica Syndrome. A Case Report. American Journal of Sports Medicine, 20, 92-94. https://doi.org/10.1177/036354659202000121

[2] Hardaker, W.T., Whipple, T.L. and Bassett, F.H. (1980) Diagnosis and Treatment of the Plica Syndrome of the Knee. Journal of Bone and Joint Surgery. American Volume, 62-A, 221-225. https://doi.org/10.2106/00004623-198062020-00008

[3] Kobayashi, Y., Murakami, R., Tajima, H., Yamamoto, K., Ichikawa, T., Mase, Y. and Kumazaki, T. (2001) Direct MR Arthrography of Plica Synovialis Mediopatellaris. Acta Radiologica, 42, 286-290. https://doi.org/10.1080/028418501127346837

[4] Dupont, J.Y. (1997) Synovial Plicae of the Knee. Controversies and Review. Clinics in Sports Medicine, 16, 87-122. https://doi.org/10.1016/S0278-5919(05)70009-0

[5] Bough, B.W. and Regan, B.F. (1985) Medial and Lateral Synovial Plicae of the Knee: Pathological Significance, Diagnosis and Treatment by Arthroscopic Surgery. Irish Medical Journal, 78, 279-282.

[6] Dorchak, J.D., Barrack, R.L., Kneisl, J.S. and Alexander, A.H. (1991) Arthoroscopic Treatment of Symptomatic Synovial Plica of the Knee. Long-Term Followup. American Journal of Sports Medicine, 19, 503-507. https://doi.org/10.1177/036354659101900515

[7] Kim, S.J. and Choe, W.S. (1997) Arthoroscopic Findings of the Synovial Plicae of the Knee. Arthoroscopy, 13, 33-41. https://doi.org/10.1016/S0749-8063(97)90207-3

[8] Older, J., Hanspal, R. and Cardoso, T. (1986) The Medial Shelf: An Anatomical, Clinical and Pathological Study. In Trickey, E.L. and Hertel, P., Eds., Surgery and Arthoscopy of the Knee, Springer-Verlag, Berlin, 82-86. 
https://doi.org/10.1007/978-3-642-71022-3_34

[9] Tearse, D.S., Clancy, W.G. and Gersoff, W.K. (1988) The Symptomatic Lateral Synovial Plica of the Knee. Communication, 7 th Annual Meeting of the Arthroscopy Association of North America, Washington DC.

[10] Kim, Y.M., Kim, S.J., Hwang, D.S., Shin, H.D., Yang, J.Y. and Kwon, S.T. (2007) Inferolateral Parapatellar Synovial Fold Causing Patellofemoral Impingement in Both Knee Joints. Arthroscopy, 23, 563.e1-563.e4. https://doi.org/10.1016/j.arthro.2005.12.041

[11] Broom, M.J. and Fulkerson, J.P. (1986) The Plica Syndrome: A New Perspective. Orthopedic Clinics of North America, 17, 279-281.

[12] Schindler, O.S. (2014) “The Sneaky Plica” Revisited: Morphology, Pathophysiology and Treatment of Synovial Plicae of the Knee. Knee Surgery, Sports Traumatology, Arthroscopy, 22, 247-262. https://doi.org/10.1007/s00167-013-2368-4

Submit or recommend next manuscript to SCIRP and we will provide best service for you:

Accepting pre-submission inquiries through Email, Facebook, LinkedIn, Twitter, etc. A wide selection of journals (inclusive of 9 subjects, more than 200 journals)

Providing 24-hour high-quality service

User-friendly online submission system

Fair and swift peer-review system

Efficient typesetting and proofreading procedure

Display of the result of downloads and visits, as well as the number of cited articles

Maximum dissemination of your research work

Submit your manuscript at: http://papersubmission.scirp.org/

Or contact ojo@scirp.org 